\title{
An Integrated Young Interferometer Based on UV-Imprinted Polymer Waveguides for Label-Free Biosensing Applications
}

\section{Wang \\ meng@ee.oulu.fi \\ J. Hiltunen \\ C. Liedert \\ L. Hakalahti}

R. Myllylä
Optoelectronics and Measurement Techniques Laboratory, University of Oulu, Finland

VTT Technical Research Center of Finland, Oulu, Finland

VTT Technical Research Center of Finland, Oulu, Finland

VTT Technical Research Center of Finland, Oulu, Finland

We demonstrate a polymer-rib waveguide Young interferometer sensor fabricated by UV imprinting. An inverted-rib waveguide structure was utilized to simplify the fabrication process. In this configuration, grooves were formed on the undercladding layer by UV imprinting and the core material was spin coated on top to fill the grooves. A glucose-water solution was used to characterize the sensor response against bulk refractive index changes. Recorded sensing responses show a linear correspondence with the refractive index changes of glucose solutions with a detection limit of about $10^{-5}$. To verify the polymer sensor's biosensing capability, an immunoassay was performed with c-reactive proteins (CRP) and human CRP-specific antibodies adsorbed on the waveguide surface. A CRP solution in a PBS (phosphate buffered saline) buffer with a concentration of $2 \mu \mathrm{g} / \mathrm{ml}(16 \mathrm{nM})$ produced a marked response, exceeding the noise level over two hundred times. Based on these values, a detection limit of about $2.4 \mathrm{pg} / \mathrm{mm}^{2}$ was determined for surface sensing of molecular adsorption. With the proposed waveguide configuration, the fabrication of polymer sensors can be ultimately transferred to roll-to-roll mass production to produce low-cost disposable sensors.

[DOI: http://dx.doi.org/10.2971/jeos.2012.12019]

Keywords: polymer waveguide, planar waveguide biosensors, UV-imprinting lithography, Young interferometer

\section{INTRODUCTION}

Applying optical methods to label-free detection of biomolecular binding interactions has become increasingly popular. Among the advantages of label-free optical biosensors are real-time detection ability and rapid detection process. In addition, they are free from disturbances from conjugated fluorescent or radioactive labels [1]. Label-free biosensors often use optical planar waveguide transducers, which offer such advantages as robustness, reproducibility, reliability, high integration capability and multi-analyte detection ability [2]. Many label-free optical biosensor configurations are built on the platform of planar waveguides, including surface plasma resonance (SPR) [3, 4], ring resonators [5]-[7], grating coupler sensors [8,9] and interferometers [10]-[13]. Guided-wave dielectric sensors utilize an evanescent field to probe refractive index changes on the waveguide surface. A typical penetration depth of a few hundred nanometres [14] provides an adequate coverage for most types of small biomolecules.

Interferometric sensors typically consist of closely placed reference and sensing arms, offering inherent background compensation against environmental disturbances, such as temperature fluctuations. This technique is considered as one of the most sensitive for optical sensing [15]. A Young interfer- ometer generates a complete periodic intensity pattern and allows determination of the absolute sign of the induced phase change, while producing a linear correlation between the spatial change of the interference pattern and the induced phase change [16].

Fabrication of conventional inorganic dielectric-based rib waveguides often requires expensive patterning steps and reactive ion etching (RIE) [17] to define the ridge. As a result, their fabrication is costly and time-consuming. With the fast development of the telecoms industry, polymers have become important component materials for waveguide manufacture. Among their good optical properties are, most importantly, high optical transmittance, versatile processability at relatively low temperatures and potential for low-cost production. Using an imprinting technique allows polymers to be moulded easily into structures containing features down to tens of nanometres [18].

The motivation of this work was to develop cost-effective disposable waveguide-based biosensors compatible with highvolume production. With UV imprinting, patterns can be replicated rapidly at room temperature by pressing a mould 


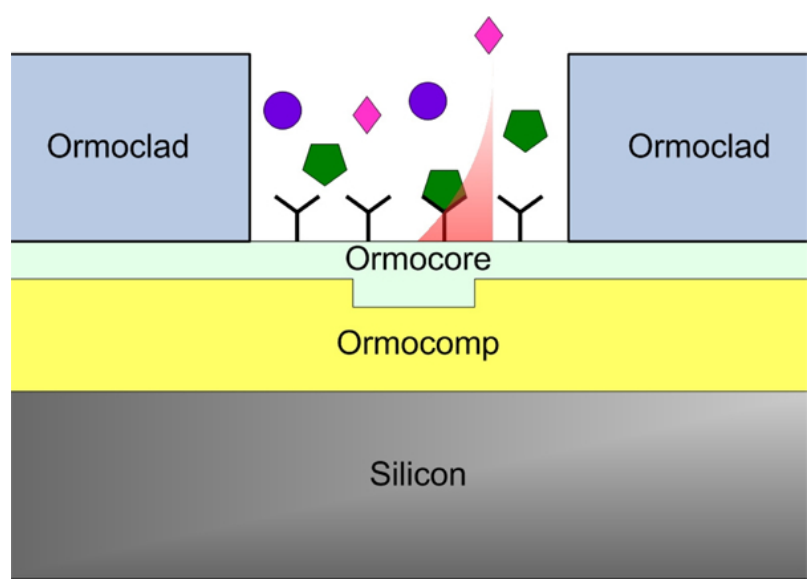

FIC. 1 Schematic view of the inverted-rib waveguide with a sensing window opened above it. Antibodies immobilized on the sensing waveguide are used for capturing target biomolecules in analytes.

onto a UV-sensitive resin and curing it under UV light. For the fabrication of an inverted-rib waveguide-based Young interferometer, we chose to pattern the undercladding of the waveguide and spin coat the polymeric core material above the patterned grooves. Based on our previous research [19, 20], the mould used for UV imprinting of Young's interferometer was also made from polymers, because they allow a fast prototyping process with a short turn-around time. The formed inverted-rib waveguide-based interferometer is characterized by different concentrations of glucose-water solutions in order to determine their sensitivity for homogeneous sensing of refractive index. The sensing ability of biomolecule interactions is validated by monitoring the specific binding of CRP to CRP antibodies adsorbed on the polymer waveguide surface.

\section{MATERIALS AND METHODS}

\subsection{Sensor fabrication by UV imprinting lithography}

The Young polymer-interferometer sensor was fabricated with a series of commercial inorganic-organic hybrid materials, Ormocers (Micro resist technology $\mathrm{GmbH}$, Germany). The series of hybrid materials behaves like negative tone photoresists, but have a better thermal and chemical stability, due to inorganic networks of Si-O-Si formed backbone [21]. This type of structure is highly transparent above the wavelength of $400 \mathrm{~nm}$ and up to the near-infrared region. In the inverted-rib waveguide-based sensor shown in Figure 1, the core was made of Ormocore with a refractive index of 1.553, while Ormocomp with a refractive index of 1.520 was used as undercladding material. As uppercladding, we used Ormoclad with a refractive index of 1.536, with a sensing window opened above the sensing waveguide. The refractive index of the core and cladding materials were determined at the wavelength of $633 \mathrm{~nm}$ with a Metricon prism coupler. For mechanical support, the whole polymer waveguide structure was built on a silicon wafer.

The details for the imprint mould and actual waveguide fabrication are described in [21]. Fabrication of the inverted-rib waveguide-based interferometer is an etch-free process. Both
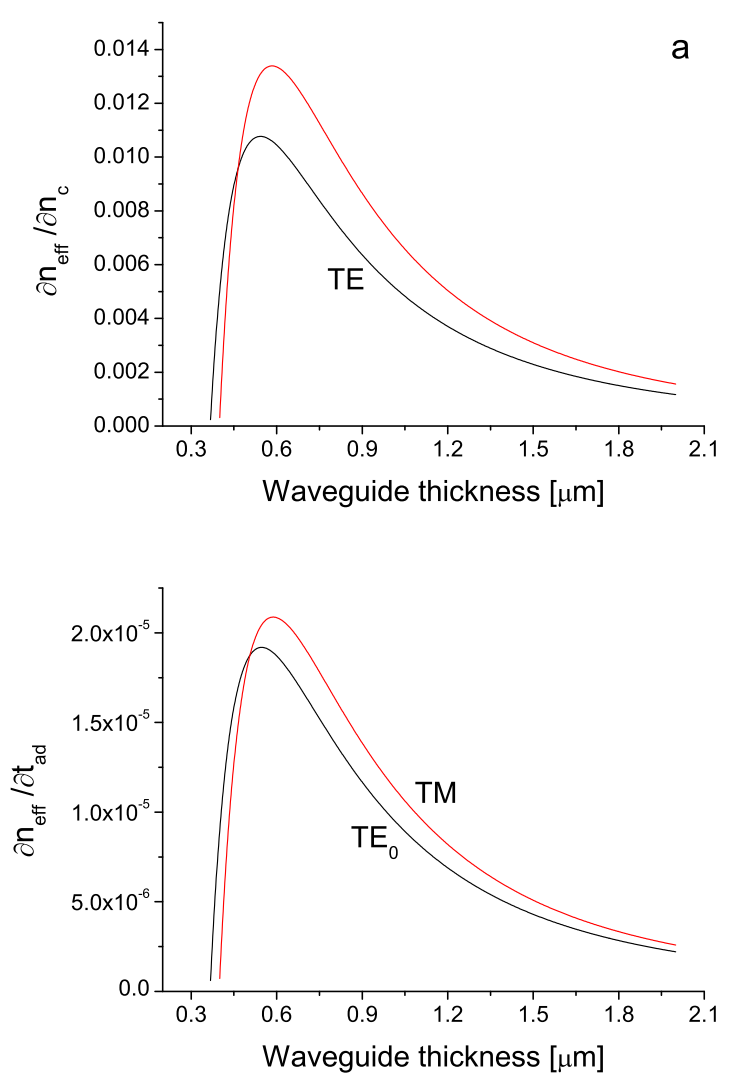

FIG. 2 Sensitivity of a) homogeneous bulk refractive index change and b) surface adsorption of biomolecules, plotted as a function of the waveguide thickness.

the imprinted mould and the inverted-rib waveguide are patterned without involving a RIE procedure which might cause additional surface roughness leading to increased scattering loss. This simple process flow provides an opportunity for fast prototyping of waveguide-based devices with a short turnaround time, high-quality performance and a potential opportunity to employ the high-volume roll-to-roll printing technique. In brief, the imprinting mould was pressed against the undercladding (Ormocomp) of the waveguide to form grooves in it. Ormocore was then spin coated on the patterned undercladding to fill up the grooves. Finally, a thick layer of Ormoclad was coated and a sensing window was opened above the sensing waveguide through a photolithographic step to form the sensing window.

The sensitivity of a waveguide-based sensor depends on the optical field distribution in the waveguide. The greater the fraction of evanescent field at the interface of the waveguide and the analyte in the sensing medium, the higher the sensitivity. Distribution of the optical field in a waveguide depends on the thickness of the waveguide and the refractive index contrast between the core and cladding materials. With the selected materials Ormocore and Ormocomp for the core and cladding, we studied the sensitivity of the waveguide sensor while varying its thickness according the method reported by Tiefenthaler et al. [22]. Simulations were conducted in the $\mathrm{TE}_{0}$ and $\mathrm{TM}_{0}$ modes to examine both homogenous sensing of bulk refractive changes and surface sensing of molecule adsorption. Figures 2(a) and 2(b) plot the sensitivity $\partial n_{e f f} / \partial n_{c}$ over the refractive index change of the cover medium $n_{c}$ and 


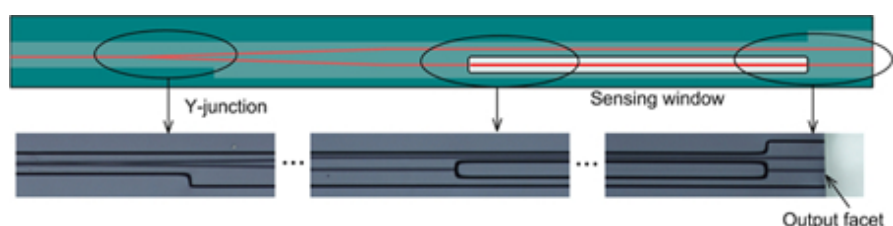

FIG. 3 Top view images of the rib-waveguide Young interferometer taken with a microscope, with a schematic illustration shown above.

the sensitivity $\partial n_{e f f} / \partial t_{a d}$ over the adlayer thickness $t_{a d}$ as a function of waveguide thickness.

Presented in Figure 2(b), the simulation of surface sensing was carried out with $n_{a d}=1.45$. As indicated by both Figures 2(a) and 2(b), highest sensitivity was achieved at a waveguide thickness of $580 \mathrm{~nm}$. Since it is easier from the processing viewpoint to construct waveguides with an overall thickness of around $1 \mu \mathrm{m}$, the TM coupling mode was chosen to enable a higher detection sensitivity. The moulded groove measures $400 \mathrm{~nm}$ in thickness and $2 \mu \mathrm{m}$ in width, determined by a Dektak profilometer. The thickness of the slab/residual layer above the filled groove was about $500 \mathrm{~nm}$. This results in an overall thickness of $\sim 900 \mathrm{~nm}$, which gives a sensitivity of 0.0087 for homogeneous sensing and $1.4 \times 10^{-5} \mathrm{~nm}^{-1}$ for the surface sensing of molecule adsorption. A schematic view of the cross-section of the sensing waveguide is shown in Figure 1.

Figure 3 shows a schematic picture and corresponding microscope images of the Young interferometer sensor. Omitted from the picture are the middle parts of the interferometer, due to the long structure of the waveguide. The length of the interferometer from the input end facet to the output is $20 \mathrm{~mm}$, while the sensing window has the length of $10 \mathrm{~mm}$. The distance between the sensing and referencing waveguide is $50 \mu \mathrm{m}$, with the reference waveguide completely covered under the Ormoclad cladding.

\subsection{Device characterization}

A linear polarized He-Ne laser $(632.8 \mathrm{~nm}, 5 \mathrm{~mW})$ was used both for sensor device characterization and the actual sensing measurements. A linear polarizer was placed in front of the laser to enable excitation in the TM polarized mode. Using the end-fire coupling method, incident light was coupled into the waveguide through a $60 \mathrm{x}$ objective lens. At the output facet of the interferometer, a 40x objective lens collected and magnified the fringe pattern image. This image was then captured by a CMOS camera with a resolution of $1280 \times 1024$. Neither the setup for device characterization nor the following experiments contained a temperature controlling device.

With the Fimmwave commercial software package (Photon Design Inc.), we simulated the supported mode in the fabricated rib-waveguide interferometer in the sensing window and in areas covered by the overcladding layer. In this simulation, we assumed that the sensing window was filled with water having a refractive index of 1.333. Figure 4 shows the simulated TM modes in the rib waveguide with a dimension of $2 \mu \mathrm{m} \times 0.9 \mu \mathrm{m}$, consisting of a $0.4 \mu \mathrm{m}$ rib and a $0.5 \mu \mathrm{m}$ slab.
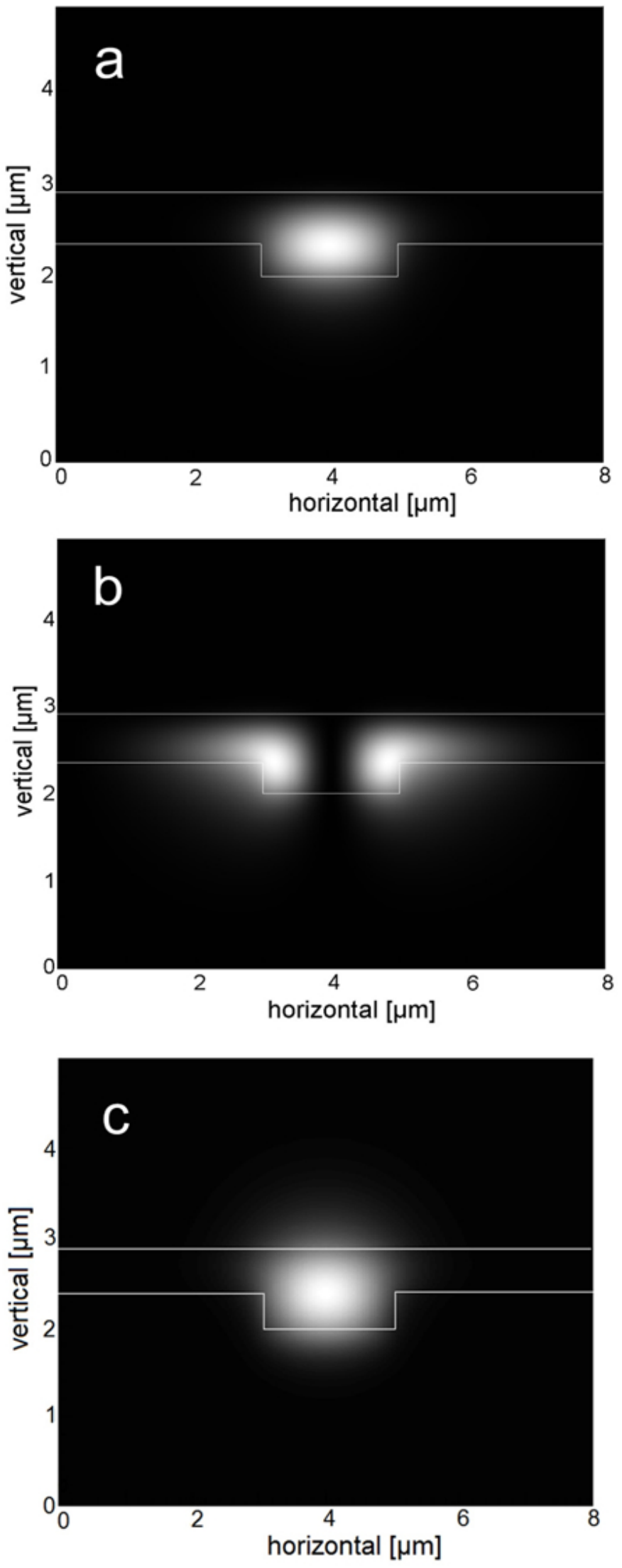

FIG. 4 Simulated mode profiles of an inverted-rib waveguide a) fundamental $T_{0}$ mode in the sensing window; $b$ ) first order $\mathrm{TM}_{1}$ mode in the sensing window; $c$ ) fundamental $\mathrm{TM}_{0}$ mode in the waveguide covered with Ormoclad cladding.

Two supported TM modes exist in the fabricated rib waveguide at $633 \mathrm{~nm}$. However, outside the sensing area, i.e., areas 


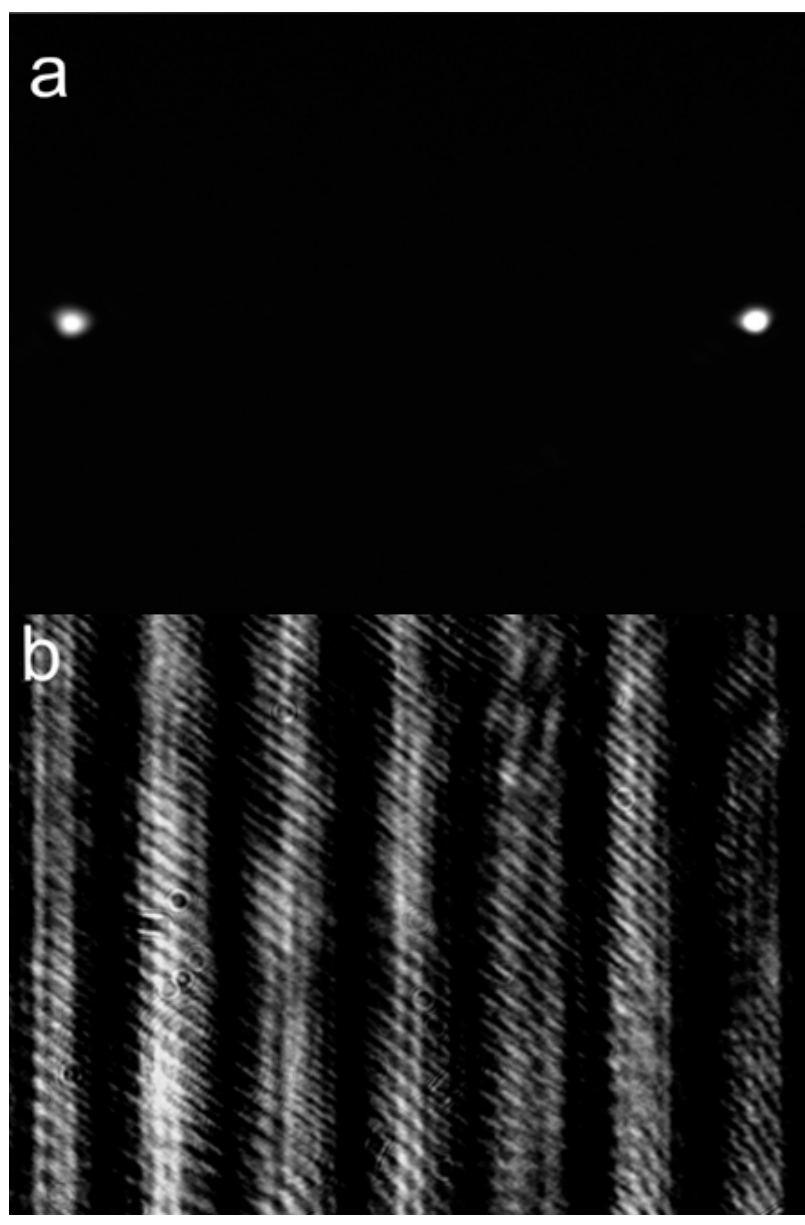

FIG. 5 Images captured by CMOS camera of a) output modes at the end facet of the interferometer; b) the generated fringe pattern.

with overcladding, only the fundamental mode is capable of propagating. Therefore, the first order mode was filtered away outside the sensing window, where the cladding consists of Ormoclad with a higher refractive index. This validates the assumption of single-mode operation.

Figure 5(a) shows the propagation mode at the output facet of the sensor chip. Two localized spots can be observed, confirming proper waveguide operation. Figure 5(b) shows the corresponding interference pattern. In this image, the imperfection in the fringe pattern was caused by stray light interference. The formed interference pattern was used in the measurements described below.

Polymeric materials, due to their molecular structure, are permeable to water vapour and able to absorb water molecules to a certain extent. When water molecules are absorbed into the polymer interferometer, the refractive index of the polymer material changes, causing a spatial shift in the fringe pattern. Since the absorption of water molecules saturates exponentially against time, the observed fringe pattern movement slows down. By immersing the sensing window in deionized water, we established a stabilization baseline for the fabricated polymer waveguide sensor, which was found to be about two hours. Consequently, later experiments started only about two hours after the sensing window had been covered in a buffer solution.

\subsection{Analyte preparation}

For homogeneous refractive index sensing, glucose solutions (D-glucose, Sigma-Aldrich), diluted with deionized water to $0.02 \%, 0.04 \%, 0.06 \%, 0.08 \%, 0.1 \%, 0.6 \%$ and $1.0 \% \mathrm{w} / \mathrm{v}$, were first prepared to calibrate the sensor. Between each measurement, deionized water was used as a buffer solution to flush the sensing window in order to bring the sensor response to the baseline. In surface sensing of molecular binding interaction, on the other hand, a PBS buffer $\left(10 \mathrm{mM} \mathrm{Na} 2 \mathrm{HPO}_{4}\right.$, $1.8 \mathrm{mM} \mathrm{NaH} \mathrm{PO}_{4}, 0.1 \mathrm{M} \mathrm{NaCl}, 2.7 \mathrm{mM} \mathrm{KCl} \mathrm{pH} \mathrm{7.4))} \mathrm{was}$ used for all solutions. As analyte, we used $2 \mu \mathrm{g} / \mathrm{ml}(16 \mathrm{nM})$ of CRP antigen (Schripps Laboratories, San Diego, CA, USA), since this concentration is utilized to predict mildly elevated risk of a cardiac event [23]. Monoclonal anti-human C-reactive protein antibodies (Medix Biochemica) with a concentration of $0.5 \mathrm{mg} / \mathrm{ml}$ were used to capture CRP antigens. To block the sensing surface from non-specific binding, $2 \mathrm{mg} / \mathrm{ml}$ of BSA (Sigma-Aldrich) was allowed to flow through the sensing window, before the injection of CRP. Moreover, $0.5 \mathrm{mg} / \mathrm{ml}$ of mouse IgG (Jackson ImmunoResearch Laboratories, Inc) served as negative control to show that no significant unspecific binding occurred.

\section{EXPERIMENT AND DISCUSSION}

In our experiments, carried out in an aqueous environment, a continuous flow syringe pump was used to deliver the analyte onto the sensing window through a flow cell attached above the sensor chip. A silicone-based gasket having an opening window with a volume of $10 \mu \mathrm{l}$ was placed between the sensing chip and the flow cell to prevent leakage of the analyte. Fringe pattern images were captured at a rate of $1 \mathrm{~Hz}$, and the phase of each image was extracted using a two-dimensional Fast Fourier Transform in the Matlab environment.

\subsection{Homogeneous sensing}

In homogeneous sensing, the liquid sample above the sensing window serves as cladding material for the waveguide. When the liquid is changed, the sensor records it as a change in the homogeneous refractive index of the waveguide cladding. It is assumed that no molecule adsorption occurs on the surface of the sensing waveguide.

The measurement started by pumping deionized water through the sensing window. After baseline stabilization, glucose-water solutions were applied, starting from the lowest concentration, i.e., $0.02 \% \mathrm{w} / \mathrm{v}$. At the beginning and end of every measurement, the sensing window was flushed with deionized water. Each measurement circle took 5 minutes. To provide a clear view of the sensing responses, Figures 6(a) and 6(a) plot the phase responses against time for glucose solutions with lower and higher concentrations. Phase changes after low-pass filtering are plotted above the original phase responses.

Effective refractive index changes can be derived from the 

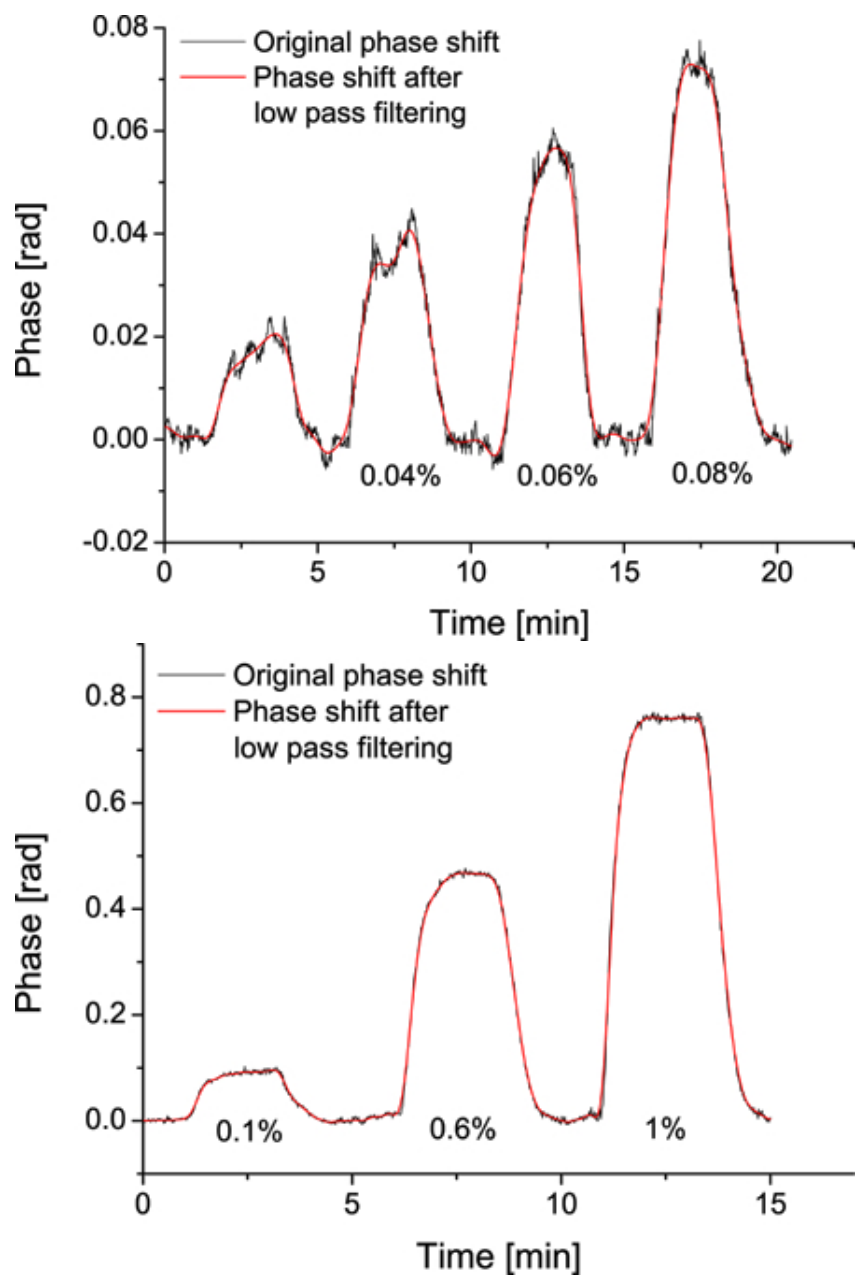

FIG. 6 Extracted phase of the fringe pattern plotted against time when applying a) low and b) high concentrations of glucose-deionized water solutions to the sensor window. Phase responses after low-pass filtering are plotted on top of the original data.

phase responses of each glucose sample using Eq. (1)

$$
\Delta \varphi=\frac{2 \pi}{\lambda_{0}} L \cdot \Delta n_{e f f}
$$

where $\Delta \phi$ is the phase change in radian, $\lambda_{0}$ is the vacuum wavelength of the monochromatic light source, and $L$ is the interaction length, which was $6 \mathrm{~mm}$ in the glucose measurements. The derived $\Delta n_{e f f}$ values are plotted in Figure 7 with respect to the actual refractive index change obtained from the concentration of the analyte.

In principle, the linear fit of effective refractive index changes is a calibration curve, showing a linear correspondence between the stimulating signal $\Delta n_{C}$ and the sensor response $\Delta n_{\text {eff }}$. This linear regression can be used to extract the actual sensitivity of the measured interferometer sensor. According to Eq. (2), the slope of the linear regression is the actual sensitivity of bulk refractive index change $\partial n_{e f f} / \partial n_{C}$

$$
\Delta n_{e f f}=\frac{\partial n_{e f f}}{\partial n_{c}} \Delta n_{c}
$$

where $\Delta n_{e f f}$ and $\Delta n_{C}$ are the effective and analyte refractive index changes. As the theoretically calculated sensitivity is 0.0087 and the measured sensitivity 0.0086 , the two figures show good agreement. To estimate the detection limit for

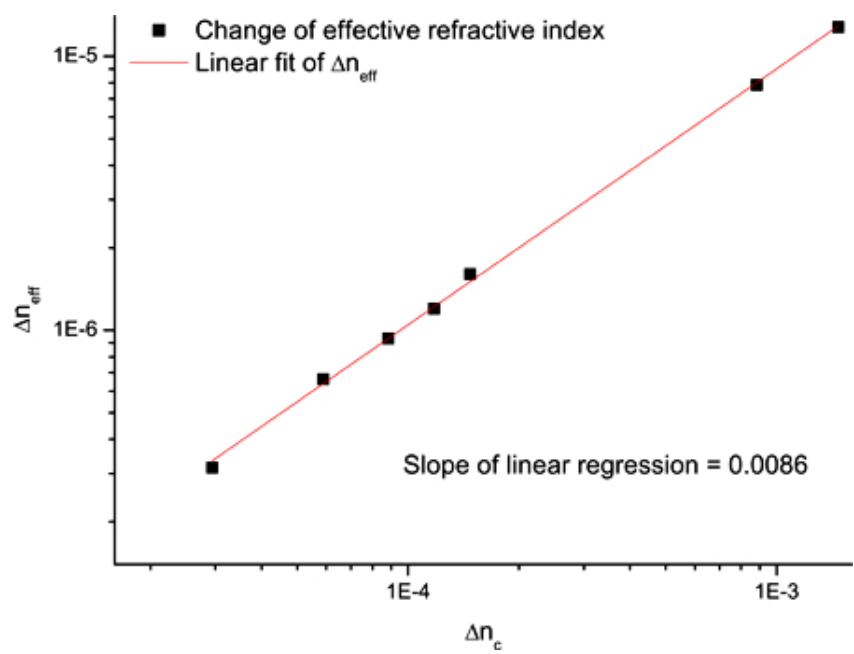

FIG. 7 Effective refractive index change plotted against actual refractive index change of glucose solutions.

homogeneous sensing, we monitored the baseline of the interferometer sensor for 5 minutes. Its noise level, defined as the standard deviation of the baseline, was around $0.002 \mathrm{rad}$. The noise present in the baseline could be caused by vibration, laser instability or air and temperature fluctuations. Since the detection limit is considered to be 3 times the noise level, we get a value of 0.006 rad for the phase response. Thus, the minimum detectable refractive index change of the analyte is $1.2 \times 10^{-5}$.

\subsection{Surface sensing of binding interaction of CRP molecules}

To demonstrate the surface sensing functionality of the polymer biosensor, a CRP immunoassay was performed. The experiment started by flushing the PBS buffer through the sensing window. After baseline stabilization, the surface was activated by pumping CRP antibodies through the sensing window for one hour. Figure 8(a) plots the phase shift of the interferogram as a function of time. When the CRP antibodies passed through the sensing waveguide, those that flowed near the sensing surface were adsorbed non-specifically on the surface of the hydrophobic Ormocore waveguide. Considerably time was spent on increasing the probability of this event. We can see in Figure 8(a) that the phase change tended to stabilize after about one hour. At that point, the PBS buffer was flushed for 20 minutes to wash away loosely attached and excessive CRP antibodies. Subsequently, BSA was injected to block any free spots where CRP could bind unspecifically to the waveguide surface. Taking into account the theoretical sensitivity of surface sensing, the actual growth of the adlayer can be calculated with the following Equation:

$$
\Delta t_{a d}=\Delta n_{e f f} / \frac{\partial n_{e f f}}{\partial t_{a d}}
$$

The corresponding surface mass density may then be obtained with De Feijter's Formula

$$
\Gamma=\Delta t_{a d} \frac{n_{a d}-n_{c}}{d n / d c},
$$

where $d n / d c$ is the refractive index increment of the protein solution. We used a value of $0.18 \mathrm{~cm}^{3} / \mathrm{g}$ for $d n / d c$, a value com- 

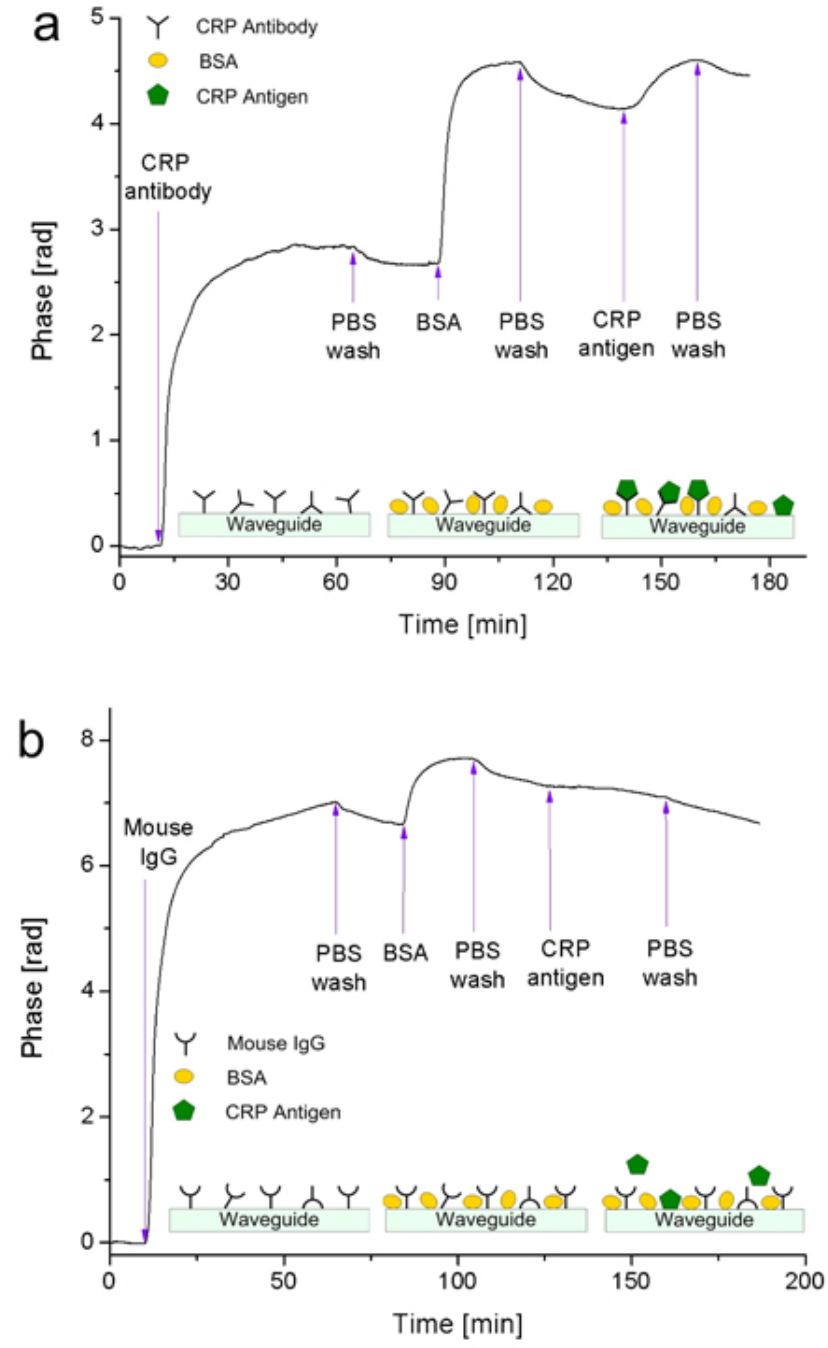

FIG. 8 Phase response in the CRP affinity experiment. CRP antibodies were used as primary biomolecules adsorbed directly on the sensing surface to capture CRP antigens; b) Phase response of the negative control. Mouse IgGs were used as primary biomolecules adsorbed directly on the sensing surface.

monly used for protein adsorption. After rinsing with PBS, $2 \mu \mathrm{g} / \mathrm{ml} \mathrm{CRP}$ antigen was pumped through the sensing window. A clear rise in the phase response is visible in Figure 8(a), indicating attachment of CRP antigens to the sensing surface. This phase increase, measured 30 minutes after the injection of CRP antigens, was about $0.46 \mathrm{rad}$. To prove that the phase change was caused by specific affinity binding between CRP antibodies and antigens, an identical experiment was conducted, the only difference being that mouse $\operatorname{IgG}$ served as receptor instead of CRP antibodies. As seen in Figure 8(b), CRP does not increase the phase response in the negative control experiment, thereby indicating that CRP does not bind to the surface in the absence of specific antibodies. Since the antibodies were not covalently attached to the sensing surface, they could detach from it during the measurement. A close look at the phase response in Figure 8(b) indicates that, when CRP antigens were injected, the slope of the phase response became less steep than that at the previous PBS washing. This could be explained by minor non-specific binding and refractive index mismatching, changing the direction of the slope of the phase response. Non-specific binding sites could be created when antibodies detach from the sensing surface.
Based on the phase plots in Figures 8(a) and 8(b), the phase change and estimated surface mass density of each injection step is listed in Table 1. It can be seen that the surface coverage of primary antibodies varies, even though their concentration and adsorption time remain the same. The reason for this might be the different affinity of these two antibodies to the hydrophobic sensing surface. After the injection of BSA molecules, the uncovered sensing surface was blocked. The resultant surface mass densities were almost the same for the CRP immunoassay and the negative control. This indicates good monolayer coverage on the sensing surfaces in both experiments.

Comparing the phase response of the negative control in Figure $8(\mathrm{~b})$ with the CRP affinity binding in Figure 8(a), we can conclude that the rise in Figure 8(a) after the injection of CRP antigens was mostly due to affinity binding between CRP antibodies and CRP antigens. Taking account of the theoretical surface sensing sensitivity, a rise of 0.46 rad corresponds to an effective increase of $0.55 \mathrm{~nm}$ of the adlayer. Using De Feijter's Formula, the estimated surface mass density is $350 \mathrm{pg} / \mathrm{mm}^{2}$. The relatively small surface mass coverage is presumably caused by the random orientation of nonspecifically adsorbed CRP antibodies on the sensing surface; no binding sites were available for capturing antibodies. An amplification of the sensing response could be achieved by controlling the orientation of primary antibodies by a surface immobilization protocol. With this protocol, primary antibodies tend to be covalently bonded to the sensing surface, which would improve the detaching problem. With the current experimental process, for $2 \mu \mathrm{g} / \mathrm{ml}(16 \mathrm{nM}) \mathrm{CRP}$ antigen, the fabricated sensor produced a phase change of $0.46 \mathrm{rad}$, which exceeds the noise level of 0.002 rad by 230 times. Assuming that the full coverage of a closely packed monolayer has a surface mass density of $5.6 \mathrm{ng} / \mathrm{mm}^{2}$, and taking into account the noise level, the developed polymer waveguide sensor should be able to detect a mass density of $2.4 \mathrm{pg} / \mathrm{mm}^{2}$ for biomolecules adsorbed on the sensing surface.

\section{CONCLUSION}

We developed a rib-waveguide Young interferometer biosensor based on polymeric materials with inverted-rib waveguide geometry. Utilizing UV-imprinting lithography the inverted-rib interferometer can be fabricated simply through two steps: patterning/imprinting the undercladding of the waveguide and spin coating the core. Absence of etching steps eliminates the risk of increasing the roughness of the waveguide surface, which might cause additional scattering loss. Measuring $2 \mu \mathrm{m} \times 0.9 \mu \mathrm{m}$, the resultant rib waveguide was operated in the single mode at $632.8 \mathrm{~nm}$, in accordance with simulations and actual waveguide characterization. The functionality of the fabricated polymer waveguide interferometer sensor was demonstrated with both homogeneous and surface-sensing mechanisms. For bulk refractive index sensing using glucose-water solutions, a detection limit of $10^{-5}$ RIU was found. For surface sensing of molecular adsorption, a surface mass density detection limit of $2.4 \mathrm{pg} / \mathrm{mm}^{2}$ can be achieved. In another experiment, using a CRP immunoassay with the help of negative control, 


\begin{tabular}{|c|c|c|c|c|}
\hline & Interaction length & Primary antibodies & Primary antibodies + BSA & CRP antigens \\
\hline $\begin{array}{c}\text { CRP immunoassay } \\
\text { (Figure 8(a)) }\end{array}$ & \multirow{2}{*}{$6 \mathrm{~mm}$} & $\Delta \phi=2.8 \mathrm{rad}$ & $\Delta \phi=4.6 \mathrm{rad}$ & $\Delta \phi=0.46 \mathrm{rad}$ \\
\cline { 2 - 5 } & & $\Gamma=3.4 \mathrm{ng} / \mathrm{mm}^{2}$ & $\Gamma=5.6 \mathrm{ng} / \mathrm{mm}^{2}$ & $\Gamma=350 \mathrm{pg} / \mathrm{mm}^{2}$ \\
\hline $\begin{array}{c}\text { Negative control } \\
\text { (Figure 8(b)) }\end{array}$ & $10 \mathrm{~mm}$ & $\Delta \phi=7.0 \mathrm{rad}$ & $\Delta \phi=7.7 \mathrm{rad}$ & - \\
\cline { 2 - 5 } & $\Gamma=5.1 \mathrm{ng} / \mathrm{mm}^{2}$ & $\Gamma=5.6 \mathrm{ng} / \mathrm{mm}^{2}$ & - \\
\hline
\end{tabular}

TABLE 1 Estimated surface mass densities for each injection of biomolecules in both the CRP experiment and negative control.

- indicates that the phase response is non-detectable when applying CRP antigens.

the affinity binding of CRP antigens with a concentration of $2 \mu \mathrm{g} / \mathrm{ml}(16 \mathrm{nM})$ to CRP antibodies was detected over non-specific adsorptions. Fabricated cost-efficiently, the polymer waveguide interferometer is capable of performing bulk refractive index sensing and biosensing. With the simplified fabrication process it is a promising alternative for industrial mass production of low-cost disposable sensors.

\section{ACKN OWLEDGEMENTS}

This work was supported by Infotech Oulu Graduate School. J.H. acknowledges the Academy of Finland for support under grant number 133814 .

\section{References}

[1] H.-M. Haake, A. Schütz, and G. Gauglitz, "Label free detection of biomolecular interaction by optical sensor," Fresen. J. Anal. Chem. 366, 576-585(2000).

[2] H. Mukundan, A. Anderson, W. Grace, K. Grace, N. Hartman, J. Martinez, and B. Swanson, "Waveguide-Based Biosensors for Pathogen Detection," Sensors 9, 5783-5809 (2009).

[3] R. D. Harris, and J. S. Wilkinson, "Waveguide surface plasmon resonance sensors," Sensor. Actuat. B-Chem. 29, 261-267 (1995).

[4] J. Dostálek, J. Ctyroký, J. Homola, E. Brynda, M. Skalský, P. Nekvindová, J. Špirková, et al., "Surface plasmon resonance biosensor based on integrated optical waveguide," Sensor. Actuat. B-Chem. 76, 8-12 (2001).

[5] A. Ksendzov and Y. Lin, "Integrated optics ring-resonator sensors for protein detection," Opt. Lett. 30, 3344-3346 (2005).

[6] A. Yalçin, K. C. Popat, J. C. Aldridge, T. A. Desai, J. Hryniewicz, N. Chbouki, B. E. Little, et al., "Optical sensing of biomolecules using microring resonators," IEEE J. Sel. Top. Quant. 12, 148-154 (2006).

[7] C. Barrios, K. Gylfason, B. Sánchez, A. Griol, H. Sohlström, M. Holgado, and R. Casquel, "Slot-waveguide biochemical sensor," Opt. Lett. 32, 3080-3082 (2007).

[8] W. Lukosz, D. Clerc, M. Ph. Nellen, Ch. Stamm, P. Weiss, "Output grating couplers on planar optical waveguides as direct immunosensors," Biosens. Bioelectron. 6, 227-232. (1991).

[9] N. Kim, I.-S. Park, W.-Y. Kim, "Salmonella detection with a directbinding optical grating coupler immunosensor," Sensor. Actuat. BChem. 121, 606-615 (2007)
[10] F. Prieto, B. Sepúlveda, A. Calle, A. Llobera, C. Domínguez, and L. M Lechuga, "Integrated Mach-Zehnder interferometer based on ARROW structures for biosensor applications," Sensor. Actuat. BChem. 92, 151-158 (2003)

[11] A. Ymeti, J. S. Kanger, J. Greve, G. A. J. Besselink, P. V. Lambeck, R. Wijn, and R. G. Heideman, "Integration of microfluidics with a four-channel integrated optical Young interferometer immunosensor," Biosens. Bioelectron. 20, 1417-1421 (2005)

[12] J. Xu, D. Suarez, and D. S. Gottfried, "Detection of avian influenza virus using an interferometric biosensor," Anal. Bioanal. Chem. 389, 1193-1199 (2007).

[13] T. Zhang, P. Pathak, S. Karandikar, R. Giorno, and L. Que, "A polymer nanostructured Fabry-Perot interferometer based biosensor," Biosens. Bioelectron. 30, 128-132 (2011).

[14] F. S. Ligler, and C. R. Taitt, Optical Biosensors, Second Edition: Today and Tomorrow (Elsevier Science, München, 2008).

[15] W. Lukosz, "Principles and sensitivities of integrated optical and surface plasmon sensors for direct affinity sensing and immunosensing," Biosens. Bioelectron. 6, 215-225 (1991).

[16] G. H. Cross, Y. Ren, and N. J. Freeman, "Young's fringes from vertically integrated slab waveguides: Applications to humidity sensing," J. Appl. Phys. 86, 6483 (1999).

[17] R. G. Hunsperger, Integrated optics theory and technology (Springer, Berlin, 2009).

[18] L. J. Guo, "Nanoimprint Lithography: Methods and Material Requirements," Adv. Mater. 19, 1521-4095 (2007).

[19] J. Hiltunen, M. Hiltunen, J. Puustinen, J. Lappalainen, and P. Karioja, "Fabrication of optical waveguides by imprinting: Usage of positive tone resist as a mould for UV-curable polymer," Opt. Express 17, 22813-22822 (2009).

[20] M. Wang, J. Hiltunen, S. Uusitalo, J. Puustinen, J. Lappalainen, P. Karioja, and R. Myllylä, "Fabrication of optical inverted-rib waveguides using UV-imprinting," Microelectron. Eng. 88, 175-178 (2011).

[21] U. Streppel, P. Dannberg, C. Wächter, A. Bräuer, L. Fröhlich, R. Houbertz, and M. Popall, "New wafer-scale fabrication method for stacked optical waveguide interconnects and 3D micro-optic structures using photoresponsive (inorganic-organic hybrid) polymers," Opt. Mater. 21, 475-483 (2003).

[22] K. Tiefenthaler, and W. Lukosz, "Sensitivity of grating couplers as integrated-optical chemical sensors," J. Opt. Soc. Am. B 6, 209-220 (1989).

[23] M. B. Pepys, and G. M. Hirschfield, "C-reactive protein: a critical update," J. Clin. Invest. 111, 1805-1812 (2003). 